\title{
Arousal from sleep: The effects of the cognitive value of auditory stimuli
}

\author{
T. E. LeVERE, N. DAVIS, JEANNE MILLS, and ELLIOTT H. BERGER \\ North Carolina State University, Raleigh, Vorth Carolina 27607
}

\begin{abstract}
The present research describes the effects that different histories of positive reinforcement may have relative to the arousal produced during sleep by otherwise quite similar auditory stimuli. In general, the cognitive loading established by different reinforcement histories appears to be an effective parameter determining arousal but only within certain limitations. First, the differential effects were noted only during sleep characterized by slow-wave EEG activity and not during sleep characterized by fast-wave EEG activity. Secondly, it would appear that the amount of reinforcement is not necessarily the controlling variable. Rather, it would appear that the consistency of the occurrence of reinforcements relative to the individual's behavior is critically related to how effective the auditory stimulus is in producing arousal during sleep. These results are interpreted as supporting the contention that sleep, or at least certain types of sleep, represent a need state.
\end{abstract}

Recent investigations concerned with how an individual responds to auditory stimuli have indicated certain differences between responses during sleep as compared to responses during wakefulness (LeVere, Bartus, Morlock, \& Hart, 1973; LeVere, Davis, Mills, Berger, \& Reiter, 1976; LeVere, Morlock, Thomas, \& Hart, 1974). The general theme governing these and other similar researches (see summary by LeVere et al., 1976) is a definition of the sleep state by inquiring how an individual responds to extrinsic stimuli during this particular psychophysical condition. While not in any way denying the accomplishments of this line of investigation, it is necessary to point out that the researches have by and large been somewhat restricted relative to parameters of an extrinsic stimulus. That is, an extrinsic stimulus may be considered to have two qualitatively distinct sets of characteristics which determine an individual's response, i.e., a cluster of physical characteristics and a cluster of psychological characteristics. To attempt to investigate sleep by cataloging how an individual responds to auditory stimulation requires an appreciation of the relative influence of both these sets of characteristics. However, more often than not, contemporary investigations have ignored the psychological aspects in favor of the physical aspects. While this may produce some empirical efficiency, it does this at the cost of providing something less than complete data.

This research was supported by National Aeronautics and Space Administration Research Grant NGL 34-002-095. T. E. LeVere, N. Davis, and Jeanne Mills are members of the Neuropsychology Laboratory, Department of Psychology, and Elliott $\mathrm{H}$. Berger is a member of the Center for Acoustical Studies, Department of Mechanical and Aerospace Engineering. The authors wish to thank D. Miller for her assistance in preparing this report. Requests for reprints should be directed to: T. E. LeVere, Neuropsychology Laboratory, Department of Psychology, North Carolina State University, Raleigh, North Carolina 27607.
This is not to say that the psychological aspects of extrinsic stimuli have been considered totally inconsequential. For example, Oswald, Taylor, and Treisman (1960), Williams, Morlock, and Morlock (1966), and more recently Langford, Meddis, and Pearson (1974) have questioned the contribution of meaning as a parameter contributing to the arousal produced by particular auditory stimuli. However, in these researches, the question of meaningfulness has been approached somewhat obliquely by questioning whether instructions prior to sleep or punishment for failure to respond during sleep (Williams et al., 1966) or a coherent or incoherent tape-recorded playback of the subject's name (Oswald et al., 1960; Langford et al., 1974) are differentially arousing during different types of sleep. Cognitive value, as it may be generally related to the reinforcement history of a particular stimulus has not been investigated. The purpose of the present investigation was thus a preliminary step in determining whether differential reinforcement histories associated with relatively similar auditory stimuli might produce different levels of arousal when these stimuli were presented during sleep. Put otherwise, does the cognitive value of a particular auditory stimulus when learned during the waking state affect how an individual responds to this stimulus during sleep?

\section{METHODOLOGY}

\footnotetext{
Subjects

The subjects were 12 male college student volunteers between the ages of 18 and 22 years (mean $=19$ ). The subjects were matched insofar as possible with regard to their usual sleeping routines and environments. None of the subjects was suffering from any disability or taking any medication which might conceivably interfere with sleep. With the exception of their sleeping within our laboratory, all subjects were instructed to maintain their normal daily routines excluding, of course, the consumption of alcohol or other narcotics.
} 


\section{Apparatus and Procedure}

The bedroom used has been previously described in detail by LeVere et al. (1973). Briefly, it measured $12 \mathrm{ft}$ square $\times 8 \mathrm{ft}$ in height and was located within a larger laboratory area where the ambient noise level was less than $45 \mathrm{~dB}(\mathrm{~A})$ during the time the subject slept. The bedroom was furnished with a carpet, small dresser, and two chairs in addition to the subject's bed, speaker system, and the behavioral task used to establish the differential meaningfulness of the auditory stimuli used in the present research.

The behavioral task, shown in Figure 1, was essentially a reaction time situation in which the subject was required to respond to different auditory tones which were associated with different amounts of reward (money) payoffs. In terms of hardware, the task was a box measuring approximately $12 \mathrm{in}$. wide $\times 8$ in. deep and $3.5 \mathrm{in}$. in height. The top of the box, which was slightly inclined toward the subject, contained two response buttons centered between the left and right sides, a series of four counters to the left and, to the right of the response buttons, a vertical row of three status lights and, further to the right, a vertical row of two sets of three payoff lights. The status lights indicated whether the task was running (top light), in a hold mode (middle light), or off (bottom light). The subject kept the task running by holding the bottom response button depressed, which enabled the programming equipment to present the tones to which the subject was to respond. If the subject released the bottom button between tone presentations, the apparatus shifted to a hold mode and disenabled tone presentations. The purpose of this bottom button was to insure that each subject's response was similar, and, since the total amount of money the subject could earn was in a large part dependent upon the number of tones which were presented, all subjects were quite diligent in keeping the bottom button depressed between tone presentations. Wher a tone was presented, the subject had the option of responding or not responding during its $1.5-\mathrm{sec}$ duration. The subject responded to a tone by releasing the bottom button and, with the same hand, momentarily depressing the top response button and then returning to the bottom button. However, as will be described below, responding, in terms of pressing the top button, did not invariably lead to reinforcement but was sometimes punished by subtracting money from the subject's total. The particular effects of a particular response were indicated by the far-right payoff lights, with each indicating an amount of 1 penny. The top three lights indicated what amount of money would be added to the subject's total, while the bottom three lights indicated what amount of money would be subtracted. The payoff lights remained on for approximately .5 to $.75 \mathrm{sec}$ following each response or tone presentation in the event the subject elected

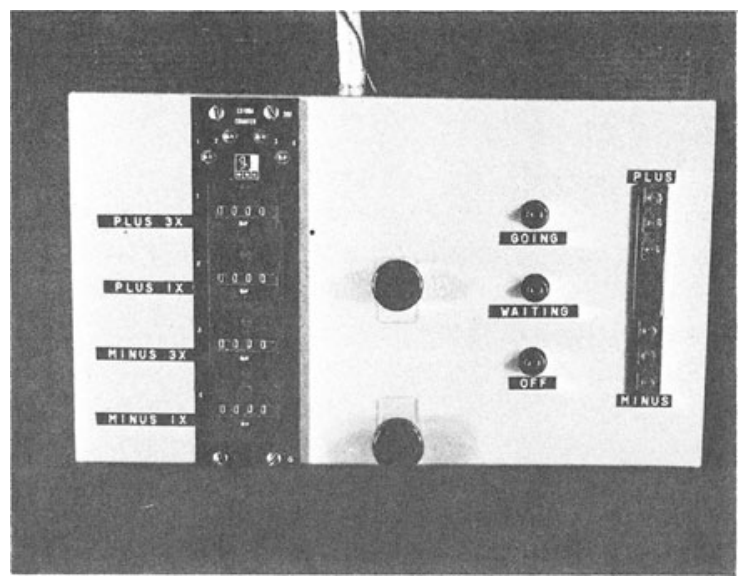

Figure 1. Photograph of the behavioral task used during the training phase of the experiment. See text for complete explanation. not to respond. The counters to the far left simply totalized the amount of money the subject earned and lost during a particular training session.

The auditory stimuli, of which there were three, were one-third octave bands centered on the frequencies of $630 \mathrm{~Hz}, 1 \mathrm{kHz}$, and $1.6 \mathrm{kHz}$. Each of the stimuli were generated by filtering random noise to exclude all frequencies save the one-third octave band centered on the frequency of interest. The intensity of these auditory stimuli was set at $80 \mathrm{~dB}$ as measured with the A-weighted network. During training, when the stimuli were used to signal different reinforcement contingencies, the duration of each was $1.5 \mathrm{sec}$. When these same stimuli were presented during sleep, each had a duration of $15 \mathrm{sec}$.

To establish a different cognitive value for these three stimuli, each subject was required to come to the laboratory for 5 days during the week preceding and on the Monday of the week that he slept in the laboratory. During these six performance sessions, the subject was required to operate the reaction-time task for two 10-min sessions (approximately 40 presentations of each stimulus during each session) and was differentially paid for responding to the three auditory stimuli. One of the stimuli was designated the "high-payoff" stimulus, and whenever the subject heard this stimulus he was rewarded with 3 cents on $66 \%$ of the presentations if he pressed the top response button. However, on $33 \%$ of the presentations, if he responded by pressing the top response button, he was penalized 3 cents. If, on the other hand, the subject did not respond to the high-payoff stimulus, he faced a penalty of 3 cents on $66 \%$ of the presentations but obtained a reward of 3 cents on $33 \%$ of the presentations. Thus, the situation presented the subject with a probability decision where responses (buttonpresses) were rewarded $66 \%$ of the time and penalized $33 \%$ of the time and not responding having just the opposite effect. The second auditory stimulus was designated as the "low-payoff" stimulus, and if the subject responded to this stimulus he was consistently rewarded 1 cent while failure to respond consistently penalized him 1 cent. The third stimulus was designated as the "no-payoff" stimulus and produced no reward and no penalization independent of the subject's response behavior. The particular auditory stimulus which was associated with a particular reward contingency was consistent for each individual subject but varied between subjects so that all three auditory stimuli were equally often associated with all three reward conditions over the duration of the experiment. While the purpose of this procedure was to establish different cognitive values to different auditory stimuli, it must be emphasized that the present research was not necessarily concerned with whether the high-payoff, low-payoff, or no-payoff condition was more meaningful to the individual subject. Rather, the experimental purpose was simply to determine whether the association of different reward conditions with different auditory stimuli could influence the arousal produced by these stimuli during sleep without any a priori predication of meaningfulness. Indeed, should the differential reward conditions be effective, any such definition might be circular, since more meaningful stimuli would be more arousing and more arousing stimuli would be more meaningful.

During the sleep phase of the present experiment, the subject's type of sleep and his responsiveness (arousal) to the auditory stimuli used in the training sessions were assessed by monopolarly recording the individual's electroencephalographic activity (EEG) with 9-mm silver cup electrodes attached to the P3 location (International 10-20 system) and referenced to the unilateral earlobe. A similar electrode attached to the outer canthus of the unilateral eye and referenced to the same ear lobe was used to detect eye movements (EOG). A final electrode was attached to the subject's neck for grounding purposes. Justification of this rather limited electrode array to analyze sleep has been previously presented by LeVere et al. $(1973,1974,1976)$. For amplification and analysis, the EEG and EOG activity were bandpass filtered between $1 \mathrm{~Hz}$ and $1 \mathrm{kHz}$ by the polygraph equipment (Grass Model 79) and again bandpass 
filtered between 1 and $35 \mathrm{~Hz}$ (Rockland Model 1022F) just prior to its input into a Digital Equipment PDP-12A computer for on-line analysis.

The PDP-12A computer was, in turn, programmed in accord with the zero-crossing frequency analysis as previously described by LeVere, Bartus, and Hart $(1972,1973)$. In general, this analysis utilizes successive 40 -sec analysis epochs and reduces the obtained frequency data by accumulating similar half-wave frequencies according to the classic band widths of delta $(0-3 \mathrm{~Hz})$, theta $(4-7 \mathrm{~Hz})$, alpha $(8-12 \mathrm{~Hz})$, and beta $(13-35 \mathrm{~Hz})$ as well as the total number of half-wave occurrences. Similarly to our previous utilization of this program, the four frequency bands were principally used to define the EEG character of the individual's sleep for stimulus presentations while the major dependent measure for determining an individual's response was the total number of half-waves occurring during analysis epoch-that is, the overall level of cortical desynchronization. As has been previously pointed out (LeVere et al., 1973, 1974), this dependent measure was used because of the direct relationship between cortical desynchronization (increases in total number of halfwaves) and arousal and the fact that this measure does not seriously violate the assumptions of standard parametric statistical procedures.

In the present research, interest was centered upon the individual's respon-iteness to the auditory stimuli during two types of sleep, i.e., sleep characterized by fast-wave EEG activity and sleep characterized by slow-wave EEG activity. The determination of fast-wave sleep and slow-wave sleep was accomplished on-line by the PDP-12A computer in accordance with the amount of time during each analysis epoch which the EEG frequency pattern showed delta, theta, alpha, or beta type activity. For example, when $20 \%$ or more of the 40 -sec analysis epoch was consumed by slow-wave delta activity, the individual was considered to be in slow-wave sleep. Fast-wave sleep was scored by the computer when the delta activity was less than $20 \%$, and additionally the alpha and beta activity were also less than $20 \%$. Wakefulness was assumed when the EEG activity fell within neither of these two categories. (Validation of this program with respect to the visual determination of wakefulness, fast-wave sleep, slow-wave sleep, and the exclusion of REM sleep has been previously presented by LeVere et al., 1974.) It should also be noted that this classification of sleep, particularly the criterion for slow-wave sleep, is compatible with the electroencephalographic criteria suggested by Rechtschaffen and Kales (Note 1). While this compatibility was intentional, it is necessary to emphasize that the present sleep classification should in no way be interpreted as indicative of sleep stages. Not only would the electrode array used in the present procedures be incompatible with an analysis of sleep in terms of sleep stages, but the data produced by any such analysis would be of relatively little use in the present procedures. The present research, as with our previous investigations, was principally interested in arousal from sleep as this is related to various types of auditory stimulation. And the dependent measure of the present procedure, that is the change in the frequency pattern of the EEG or cortical desynchronization, is not dependent upon the classification of sleep into various stages.

Each of the three nights which the subject slept in the laboratory (Tuesday, Wednesday, and Thursday) was analyzed in terms of a 6-h data acquisition period, which itself was divided into 360 40 -sec analysis epochs, each of which was separated by a 20 -sec interepoch interval. During each of these nights, each subject was presented with eight occurrences of each of the three auditory frequencies that had been used during the training procedure. Each of these stimulus presentations was $15-\mathrm{sec}$ in duration, initiated at the beginning of a 40 -sec analysis epoch, and followed a predetermined scheduled which distributed the total 24 presentations evenly over the 6-h data acquisition period. There were, however, three restrictions placed on the presentation schedule: (1) four of the eight presentations of each of the auditory stimuli were to occur when the pattern of the subject's EEG activity was dominated by fast-wave activity and four were to occur when the subject's pattern of EEG activity was dominated by slow-wave activity, (2) all stimulus presentations were to be separated by at least a 5-min interval, and (3) no stimulus presentations were to occur if rapid eye movements occupied $10 \%$ or more of either of the two immediately preceding 40 -sec analysis epochs. This latter condition represents our desire to exclude stimulus presentations during dreaming because of the possibility that the presentations might be incorporated in the dream and thereby difficult to evaluate with our dependent measures. It is, of course, realized that a single eye electrode is not entirely satisfactory for detecting uncontaminated eye movements, but for the purpose of the present research this contamination represents a somewhat conservative error. That is, the contamination of the eye-movement record when a single electrode is used principally arises from slow-wave EEG activity which thus produces an overestimation of eye movements. If the purpose of detecting eye movements is to withhold stimulus presentations during dreaming, then this overestimation will simply result in stimuli not being presented when, in fact, the stimuli could have been presented.

Within a given night, the EEG and EOG recordings were initiated immediately after the subject was settled in bed. Subsequently, the control of the experiment was under the direction of the PDP-12A computer system both for data analysis and stimulus presentations. The 6 -h data acquisition period was initiated after the computer detected five successive analysis epochs of sleep as defined above. The first stimulus presentation was scheduled for approximately $5 \mathrm{~min}$ after this point in time. It should be noted, with respect to the actual stimulus presentations, that: (1) since the auditory stimuli were presented at the initiation of the 40-sec analysis epoch, the presentation of each stimulus was based upon the two epochs immediately preceding the epoch during which it occurred; (2) since slow-wave sleep occurs predominantly during the first half of the night, presentations during this type of sleep were given priority; and (3) within a given type of sleep, all three types of auditory stimuli had to occur before a particular stimulus was repeated. When a stimulus was presented, the computer stored the EEG frequency data according to the number of half-wave occurrences within the categories of delta, theta, alpha, and beta as well as the total number of half-wave occurrences for: (1) the $40-\mathrm{sec}$ analysis epoch preceding the stimulus presentation, (2) the 40-sec analysis epoch during which the stimulus occurred, and (3) the three 40-sec analysis epochs succeeding the presentation of the stimulus. As controls for these data, eight similar groups of five analysis epochs when no stimuli had been presented were selected from the sleep period by the computer. Four of these control groupings were taken during sleep characterized by fast-wave EEG activity and four during sleep characterized by slow-wave EEG activity.

\section{RESULTS}

\section{Training Phase}

The subject's performance during the training sessions was analyzed in accord with the response strategies which might be associated with the payoff probabilities of the three reward conditions. Considering first the low-payoff stimulus, the optimal response strategy would obviously be to respond to each occurrence of the stimulus, i.e., a maximizing response strategy. To test whether the subjects were maximizing their responding to the low-payoff stimulus, the chi-square statistic was used to determine if there was a difference between the amount of reward which would be expected if the subjects responded to each occurrence of the lowpayoff stimulus and the amount of reward actually 
obtained. The results of this test indicated no significant difference between the expected amount of reward and that actually obtained $\left(x^{2}=1.97\right.$, $\mathrm{df}=1$, n.s.), indicating that the subjects were operating under a maximizing response strategy with respect to the low-payoff stimulus. So far as the no-payoff stimulus was concerned, the subjects essentially treated this stimulus like the low-payoff stimulus and responded to virtually each and every presentation of the stimulus. However, as will be seen later, this similarity during the training portion of the experiment was not reflected in the arousal produced when these two stimuli were presented during sleep.

Turning to the high-payoff reinforcement condition, the subject could opt to respond to each occurrence of the stimulus (maximizing response strategy) or respond to $66 \%$ of the stimulus presentations and withhold his response on $33 \%$ of the stimulus presentations (matching response strategy). If the subject maximized, one would expect the subject to be rewarded ( 3 cents added to his total) on $2 / 3$ of the stimulus presentations and to be punished ( 3 cents subtracted from his total) on $1 / 3$ of the stimulus presentations. If, on the other hand, the subject matched the reward probabilities, he would be rewarded on 5/9 of the stimulus presentations and punished on 4/9 of the stimulus presentations. Using the chi-square statistic to compare the expected number of rewards and punishments under each strategy with the actual obtained rewards and punishments, the data indicated that the payoff rate was significantly different from that which would be expected from the maximizing strategy $\left(x^{2}=12.57\right.$, $\mathrm{df}=1, \mathrm{p}<.01)$ but also different from the matching strategy $\left(x^{2}=60.64\right.$, df $\left.=1, p<.01\right)$. Obviously, the subjects were not consistently responding to each presentation of the high-payoff stimulus, nor were they consistently matching the reward probabilities associated with the high-payoff stimulus, but rather were somewhere in between these two response strategies. While this result may be attributed to the fact that the subjects were given only six training sessions, the important factor to remember is that the subjects did not consistently treat the highpayoff stimulus like either the low-payoff stimulus or the no-payoff stimulus, both of which produced a maximizing response strategy.

\section{Sleep Phase}

The effects of the three reinforcement conditions during sleep were averaged across the auditory frequencies with which they were associated and are presented in Figure 2. In the figure are plotted difference scores which were calculated by subtracting the number of half-waves (cortical desynchronization) occurring during the epoch just preceding a stimulus presentation from the epoch when the stimulus occurred and additionally each of the three succeeding epochs. The use of difference scores effectively normalizes the data and concentrates upon the relative arousal produced by each of the three reinforcement conditions. The data in the figure are further dichotomized on the basis of sleep type by presenting the arousal produced by stimulus presentations during fast-wave sleep on the left side of the figure and the arousal produced by stimulus presentations during slow-wave sleep on the right side of the figure. Inspection of this figure indicates that during both fast-wave sleep and slow-wave sleep, the three auditory stimuli were all capable of producing some arousal in terms of cortical desynchronization. This is, however, not terribly surprising in view of the fact that auditory stimulation independent of any specific reinforcement conditions is usually capable of disturbing sleep. However, during slowwave sleep, the figure indicates that the auditory stimuli associated with the low-payoff reinforcement condition were significantly more effective in arousing

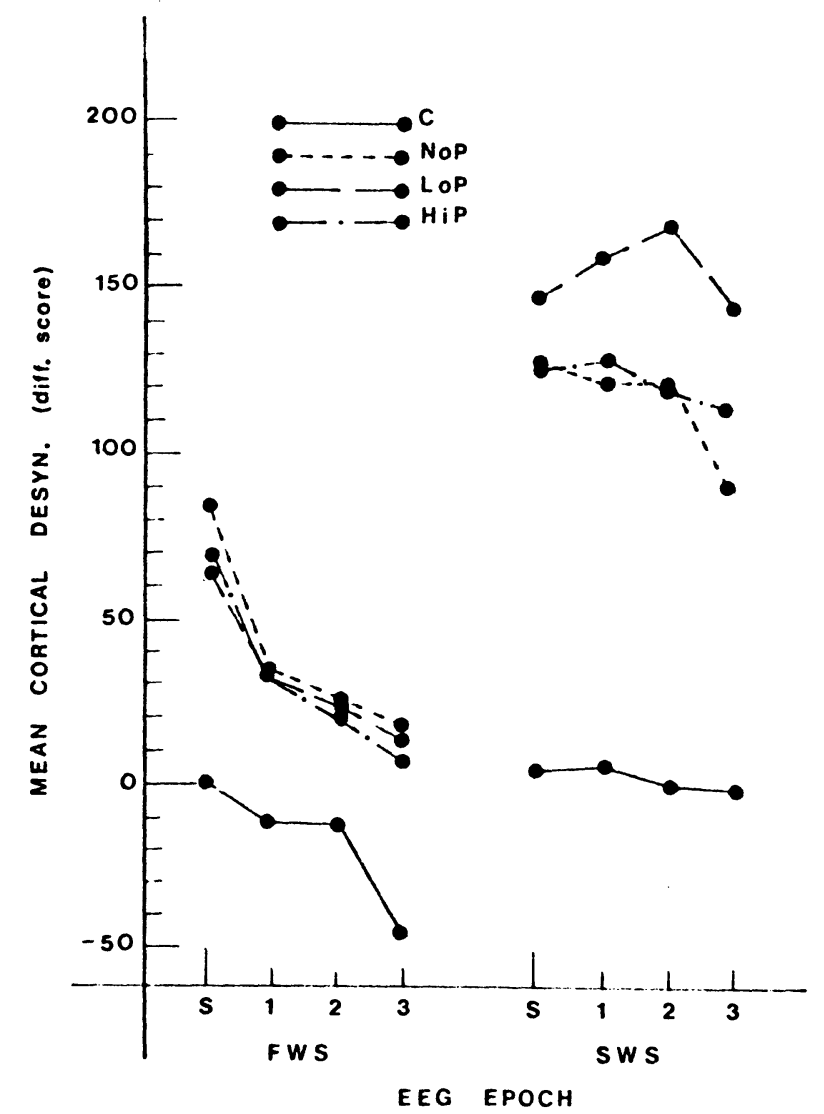

Figure 2. Mean change in cortical desynchronization (arousal) relative to the 40 -sec epoch just preceding the epoch during which the stimulus was presented. Effects of stimulus presentation during sleep characterized by fast-wave EEG activity are presented on the left, while the effects of stimulus presentations during sleep characterized by slow-wave EEG activity are presented on the right. Abbreviations: $\mathbf{C}=$ control, NoP = no-payoff reinforcement condition, $L O P=$ low-payoff reinforcement condition, HiP = high-payoff reinforcement condition. 
the individuai from sleep than the stimuli associated with the high-payoff reinforcement condition or the stimuli associated with the no-payoff reinforcement condition. During fast-wave sleep, all stimuli appeared to be about equally effective.

Statistically, an overall analysis of variance considering reinforcement condition, type of sleep, and the EEG analysis epoch with repeated measures on subjects produced two significant second-order interactions. The first was between sleep type and reinforcement condition, and the second was between sleep type and epoch. Because both interactions involve sleep type, two separate analyses of variance were performed, one on the data obtained during sleep characterized by fast-wave EEG activity and the other on the data obtained during sleep characterized by slow-wave EEG activity.

Considering first the analysis concerned with fastwave sleep, both of the main effects, reinforcement condition and analysis epoch, were significant at better than the .05 level of confidence $(F=22.59$, and $F=18.36$, 'df $=3 / 165$ in both cases). The interaction was nonsignificant. The significant main effect associated with reinforcement condition was further analyzed, using a Duncan's multiple range test, and indicated that, while the low-payoff, high-payoff, and no-payoff conditions differed significantly from the control condition, none of the three reinforcement conditions differed significantly from one another. However, this lack of difference between the auditory stimuli associated with different reinforcement conditions might not be of much interest if it was a function of a ceiling effect caused by behavioral awakening. That is, if the stimuli presented during fast-wave sleep awakened the subjects, then the cortical desynchronization would be expected to be quite similar independent of what produced the behavioral awakening. This would, of course, make total number of half-waves or cortical desynchronization a most insensitive, if not inappropriate, dependent measure. However, a ceiling effect is predicated upon the proposition that the auditory stimuli consistently produced behavioral awakening during fast-wave sleep, and this was not the case. On only $8 \%$ of the stimulus presentations during fast-wave sleep did the computer indicate that the subject was awake during any of the epochs associated with a stimulus occurrence. Thus, the similar arousal produced by the three auditory stimuli associated with the different reinforcement conditions is apparently not a function of a ceiling effect resulting from behavioral awakening. Finally, the significant main effect of analysis epoch indicates that while the auditory stimuli were effective in producing arousal, this effect was not maintained but decreased significantly over the three succeeding analysis epochs.

Turning to the analysis utilizing the data obtained during slow-wave sleep, only the main effect of reinforcement condition was significant $(F=53.93$, $\mathrm{df}=3 / 165, \mathrm{p}<.05$ ). The main effect of analysis epoch as well as the interaction were both nonsignificant. Comparisons of the three different reinforcement conditions, using again the Duncan multiple range test $(S \bar{x}=9.08, \mathrm{df}=188, \mathrm{p}<.05)$, indicated that the mean arousal associated with the low-payoff reinforcement condition was significantly different from the mean arousal associated with the highpayoff and no-payoff reinforcement conditions, which were, in turn, not different from one another. The arousal produced by each auditory stimuli was, of course, significantly different from the control condition. Finally, the fact that the main effect of analysis epoch was not significant indicated that the arousal produced by the auditory stimuli during slow-wave sleep remained relatively constant over the period of time in which the data were collected.

\section{DISCUSSION}

From a broad point of view, the present results reaffirm previous research indicating that cognitive value may contribute to the sleep disruption produced by an auditory stimulus. In this regard, the contribution of the present data is the indication that the cognitive loading need not be as heavy as the subject's personal name (Langford, et al., 1974; Oswald et al., 1960) or the threat of punishment during sleep (Williams et al., 1966). Rather, it would appear that a series of relatively protracted training sessions netting an individual between $\$ 7$ and $\$ 10$ over a period of 1 week is sufficient to differentiate quite similar auditory stimuli in terms of their arousal capacities during sleep.

Considering the data in more detail, there are two aspects which need further discussion; the differential effects of the auditory stimuli during slow-wave sleep and the greater arousal produced by the lowpayoff reinforcement condition. Firstly, the differential effectiveness of the three reinforcement conditions during slow-wave sleep corresponds to previous jesearch demonstrating the psychological distinctiveness of this type of sleep. However, in contradistinction to previous research, the present results suggest the operation, and not the absence, of certain psychological variables. For example, psychological loudness does not appear to be a relevant parameter controlling an individual's response to auditory stimuli during slow-wave sleep (LeVere et al., 1973, 1974). Additionally, material presented during slow-wave sleep is difficult to recall during wakefulness, indicating that retention is at best only minimally functional during this type of sleep (Koukkou \& Lehmann, 1968). Both of these results would suggest that slow-wave sleep may be characterized by the absence of certain psychological processes which are easily 
demonstrated in the waking individual. The present data, however, suggest that this absence is not characteristic of all psychological attributes that may be associated with a particular extrinsic stimulus. That is, cognitive value, as established by a particular reinforcement history, is surely a psychological attribute, yet it appears to be a relevant parameter in determining arousal during slow-wave sleep. What this then implies is that the absence of certain psychophysical functions and the absence of certain memory processes during slow-wave sleep should not be interpreted as indicating that it is only the physical aspects of an extrinsic stimulus which are of import to the individual during slow-wave sleep.

Turning to the differential effectiveness of the three reinforcement conditions, the arousal associated with the low-payoff reinforcement condition is, at first glance, somewhat perplexing. Again, it is necessary to emphasize that no a priori judgment of meaningfulness was associated with any of the reinforcement conditions, yet even so one might expect the high-payoff reward condition to produce greater arousal, and for at least two reasons. First, the high-payoff reinforcement condition was the only reinforcement condition which involved some degree of decision making during training. That is, the high-payoff stimulus presented the subject with the possibility of reward for both responding and not responding. The low-payoff reward condition and the no-payoff reward condition did not offer this option. The low-payoff reinforcement condition only rewarded the subject for buttonpress responses, and the no-payoff reward condition never rewarded the subject. These particular conditions would then tend to suggest that the high-payoff reinforcement condition would generate a greater degree of subjectstimulus interaction which might be expected to carry over to the sleeping state and precipitate a greater degree of arousal. Second, the high-payoff reinforcement condition was further differentiated from the other reinforcement conditions by being associated with three times the monetary reward potential. And, in fact, both of these suppositions were supported by the subjects' performance during training inasmuch as it was only. when the highpayoff stimulus was presented that the subjects withheld buttonpress responses.

Notwithstanding these arguments, the data clearly indicated that it was not the auditory stimulus associated with the high-payoff reinforcement condition which produced the greatest arousal during sleep but, rather, the stimulus associated with the low-payoff reinforcement condition. In fact, the arousal produced by the stimulus associated with the high-payoff reinforcement condition was not statistically distinguishable from the arousal which was produced by the stimulus associated with the no-payoff reinforcement condition. In view of these results, one might at first suggest that, since the subject withheld responses to the high-payoff stimulus during training, the arousal produced during sleep might be simply related to the total number of responses which the subject made to the particular auditory stimuli during training. But to espouse this sort of explanation of the increased arousal associated with the low-payoff reward condition would be to ignore the fact that during training the subject responded just as much to the stimulus associated with the no-payoff reinforcement condition which produced significantly less arousal. Alternately, it might be possible to suggest that the arousal produced during sleep might be related to the amount of money the subject obtained relative to each of the reinforcement conditions. In this case, there is a somewhat better fit to the data since the subjects averaged approximately $\$ 3.97$ for their efforts with respect to the high-payoff stimulus while averaging $\$ 4.94$ for responding to the low-payoff stimulus. However, if it was just quantity of reinforcement which determined the arousal during sleep, then the question remains as to why there was no difference between the high-payoff stimulus and the stimulus associated with the no-payoff reinforcement condition. Clearly, there must be something besides the total number of responses made to a stimulus, the amount of money earned relative to a stimulus, and the existence of reward decisions during training which is responsible for the differential arousal associated with the three reinforcement conditions.

What we would like to suggest this something might be is the nature of sleep itself. That is, we believe that the present data represent an affirmation of sleep as a need state which the individual actively attempts to satisfy, as suggested by the data related to the rebound effect and selective sleep deprivation (see Kales, 1969). From this posture, it is possible to argue that those extrinsic stimuli which carry little significance for the individual might be ignored or at least reduced in their arousal potential. It is in consideration of this that the increased arousal associated with the low-payoff stimulus may be understood. That is, it was only the low-payoff stimulus which presented the subject with consistent reward for consistent response behavior. The high-payoff stimulus sometimes did but sometimes did not, and while the no-payoff stimulus was consistent it never led to reinforcement. Thus, what we are suggesting is that those stimuli which have a greater potential for intrusion into sleep are those stimuli which have a consistent history of significance for the individual. Those stimuli which do not are reacted to, when reacted to, simply on the basis of their physical characteristics. The fact that retention may be retarded and that psychological loudness may not be relevant during slow-wave sleep is 
compatible with this sort of proposition. In both cases, it is possible to imagine that the elimination of these psychological processes is conducive to the maintenance of sleep since neither is by itself of significance to the well-being of the sleeping individual. In fact, considering sleep as a need state, the operation of these processes might even be considered detrimental since they might interfere with sleep. However, on the other hand, extrinsic stimuli which have been consistently associated with reinforcement, either positive or negative, might be of considerable import to the well-being of the sleeping individual and as such might command a degree of arousal over and above that predictable on the basis of their physical characteristics. Put otherwise, during slowwave sleep, a bird (or predator) in the hand demands more attention than two in the bush.

Whether or not the above is palatable, the fact still remains that cognitive value would appear to be an important parameter determining an individual's response during sleep. Moreover, it is apparent from the present data that this cognitive value may be established relatively rapidly through standard conditioning procedures. Whether or not it would be just as easy to eliminate this cognitive weighting through standard extinction procedures remains to be answered.

\section{REFERENCE NOTE}

1. Rechtschaffen, A., \& Kales, A. A manual of standardized terminology, techniques and scoring system for sleep stages of human subjects. U.S. Department of Health, Education and Welfare, Bethesda, Maryland, 1968.

\section{REFERENCES}

KALES, A. Sleep, physiology and pathology: A symposium. Philadelphia: Lippincott, 1969.

Koukkou, M., \& LehmanN, D. EEG and memory storage in sleep experiments with humans. Electroencephalography and Clinical Neurophysiology, 1968, 25, 455-462.

Langford, G. W., Meddis, R., \& Pearson, A. J. D. Awakening latency from sleep for meaningful and non-meaningful stimuli. Psychophysiology, 1974, 11, 1-5.

LeVERE, T. E., Bartus, R. T., \& HaRT, F. D. Electroencephalographic and behavioral effects of nocturnally occurring jet aircraft sounds. Aerospace Medicine, 1972, 43, 384-389.

LeVere, T. E., Bartus, R. T., Morlock, G. W., \& Hart, F. D. Arousal from sleep: Responsiveness to different auditory frequencies equated for loudness. Physiology and Behavior, 1973, 10, 53-57.

LeVere, T. E., Morlock, G. W., Thomas, L. P., \& Hart, F. D. Arousal from sleep: The differential effect of frequencies equated for loudness. Physiology and Behavior, 1974, 12, 573-582.

LeVere, T. E., Davis, N., Mills, J., Berger, E. H., \& Reiter, W. F. Arousal from sleep: The effects of the rise-time of auditory stimuli. Physiological Psychology, 1976, 4, 213-218.

Oswald. I., Taylor, A. M., \& Treisman, M. Discriminative responses to stimulation during human sleep. Brain, 1960, 83, 440-453.

Williams, H. L., Morlock, H. C., \& Morlock, J. V. Instrumental behavior during sleep. Psychophysiology, 1966, 2 , 208-216.
(Received for publication March 2, 1976; revision accepted April 20, 1976.) 